\title{
Vom Nationalstaat in die Europäische Union - zur europäischen Sozialisation ehemals nationalstaatlicher Politiker
}

\author{
Reinhard Bütikofer (MdEP) im Gespräch mit Joachim Jens Hesse
}

Herr Bütikofer, das Zentrum Ihrer politischen Arbeit hat sich vor nicht allzu langer Zeit von Berlin nach Brüssel und Straßburg verschoben. Hatten Sie bereits zuvor Erfahrungen mit der europäischen Politik gesammelt?

Nach einem Jahrzehnt in der Bundespolitik wechselte ich mit der Europawahl 2009 von meiner Funktion in der Grünen Bundespartei ins Europäische Parlament. Als Parlamentarier hatte ich zuletzt 1996 gearbeitet, im Stuttgarter Landtag. Mein europapolitisches Engagement konzentrierte sich, vor meiner Wahl zum MdEP, auf innerparteiliche Programmarbeit und die langjährige Mitarbeit als deutscher Vertreter in der Europäischen Grünen Partei, der grünen Parteifamilie, die mit insgesamt 46 Mitgliedsparteien aus 40 Staaten über die EU hinaus reicht.

Welche Lernprozesse als neuer Abgeordneter im Europäischen Parlament standen in den ersten zwei Jahren Ihrer Tätigkeit im Vordergrund?

Die Fraktion im Europäischen Parlament ist für mich ein deutlich weniger bedeutsamer Orientierungsraum, als das früher einmal, als MdL, die Fraktion im baden-württembergischen Landtag war oder als ich das bei MdBs in der grünen Fraktion in Berlin erlebt habe. Das liegt nicht nur an der nationalen Vielfalt. Abgeordnete auch einer kleinen Fraktion im Europäischen Parlament sind im

\footnotetext{
Anmerkung der Redaktion: Das Interview mit Reinhard Bütikofer begründet eine Reihe von Gesprächen mit Europapolitikern aus unterschiedlichen Mitgliedstaaten und Parteifamilien. Dabei geht es vor allem um den Prozess des Übergangs von der nationalstaatlichen auf die europäische politische Bühne, unter Einschluss des damit verbundenen Perspektiv- und Sichtwechsels sowie nachfolgend vertretener Politiken. Die Reihe soll damit einen Beitrag zur aktuellen Diskussionen um die Zukunft des europäischen Projekts leisten und gleichzeitig die Belastbarkeit tradierter Repräsentationsmodelle überprüfen. Auf das Gespräch mit Reinhard Bütikofer als einem ehemals prominenten Grünen-Politiker in Deutschland folgen Interviews mit sozialdemokratischen und konservativen Vertretern aus Großbritannien und Frankreich.
} 
Durchschnitt viel intensiver in die Details der Gesetzgebung eingebunden, als ich das aus Landtag oder Bundestag kenne. Daraus folgt oft ein sehr hoher Grad an Spezialisierung, aus dem sich eher eine Art „politisches Kleinunternehmertum“ ergibt als ein Agieren als Verband. Ausschussübergreifende Arbeitsgruppen, wie es sie etwa in der grünen Bundestagsfraktion gibt, stoßen daher auf erhebliche Vorbehalte, was wiederum strategische Kooperation mit Kollegen anderer Spezialisierung schwieriger macht. Auch die Fraktionshierarchie ist für die alltägliche Arbeit der Abgeordneten weniger wichtig, da man in vielen Fällen und mit hoher Selbständigkeit interfraktionelle Absprachen eingeht und Verabredungen über thematische Arbeitsteilung bei Berichten und Stellungnahmen im Ausschuss selbst klärt.

Die Rolle der Fraktion wird auch dadurch eingeschränkt, dass Abgeordnete aus verschiedenen Ländern ganz unterschiedlichen Rollenbildern folgen und unterschiedliche nationale Bezugswelten ins Auge zu fassen haben. Wenn in der Grüne/EFA-Fraktion mit 14 französischen Abgeordneten mehr grüne Mandatsträger sitzen als in der französischen Nationalversammlung, dann folgt daraus leicht, dass sie für die Repräsentation grüner Politik in Frankreich einen höheren Stellenwert genießen als die 14 deutschen grünen Abgeordneten bei sich zuhause. Französische Europaabgeordnete machen somit auch verstärkt nationale Politik und richten ihr Abstimmungsverhalten stark danach aus. Hinzu tritt, dass bei manchen wichtigen politischen Fragen der grüne politische Raum in Frankreich ganz anders gepolt ist als der in Deutschland. So ist die Europa-Fraktion weniger ein Ort gemeinsamer Meinungsbildung als des Suchens nach Platz für differierende Positionen in der Kommunikation der Gruppe. Da dem Parlament in der Gesetzgebung kein Initiativrecht zukommt, fällt das an Einigungszwang weg, was sich in Fraktionen sonst aus der Verfolgung eigener Gesetzentwürfe ergibt. Die Arbeit an Kompromissen stößt gerade dort an enge Grenzen, wo eine Mittelposition in keiner der berührten unterschiedlichen nationalen politischen Kulturen einen politischen Vorteil brächte.

Im Europäischen Parlament fällt es besonders schwer, die Basisverbindung aufrecht zu erhalten. Wegen der hohen Zahl an Sitzungswochen, die für aktive Basisarbeit ein enges Korsett vorgeben, muss man sich umso mehr anstrengen, Erfahrungen aus der Gesellschaft einzubeziehen. Ständig präsent sind dem gegenüber die unterschiedlichen Interessenvertreter. Leicht nehmen Abgeordnete und Lobbyisten sich als gemeinsame Bewohner eines weit von der Bodenstation entfernten Raumschiffs wahr. Damit sich daraus keine unangemessene Nähe entwickelt, braucht es strikte Transparenzregeln für diesen Umgang. Ich veröf- 
fentliche, sozusagen als Transparenzangebot und als Selbstschutz, im Internet fortlaufend meine Termine mit Interessenvertretern.

Viel mehr Kooperation als in deutschen Parlamenten findet sich im Europäischen Parlament zwischen einzelnen Abgeordneten unterschiedlicher Fraktionen. Das ist eine besonders attraktive Erfahrung. Wer damit geschickt umgeht, kann bisweilen auch völlig an der eigenen Fraktion vorbei wirksam intervenieren. Aber dieser Aspekt ist schon oft beschrieben worden. Hinzuzufügen ist allerdings, dass sich nach meiner Erfahrung wenig Gelegenheit zum Aufbau kontinuierlicher persönlicher Beziehungen bietet. Die Gefahr der Vereinzelung ist recht groß.

Wie prägt der neue Wirkungskreis Ihre Wahrnehmung politischer Prozesse auf der nationalen wie der europäischen Ebene?

Zunächst einmal habe ich in der neuen Funktion für meine frühere Arbeit wichtige Zugänge zur Wahrnehmung nationaler politischer Prozesse aufgegeben. Ich bin in keinem Grünen Parteigremium Mitglied. Ich habe in zwei Jahren nur einmal an einer Sitzung der Grünen Bundestagsfraktion teilgenommen. Meine Mitwirkung in informellen, innerparteilichen Netzwerken erfolgt weniger intensiv als noch vor zweieinhalb Jahren. Die systematische Beobachtung der Meinungsbildung in den grünen Landesverbänden habe ich durch eine stärkere Fokussierung auf einige wenige Regionen ersetzt. Mein Meinungsaustausch mit Bundespolitikern anderer Parteien ist deutlich zurückgegangen. Kontakte zu journalistischen Beobachtern und Kommentatoren aus der Bundespolitik sind selten geworden.

Nicht verringert, sondern ausgebaut habe ich meine Kontakte in die Wirtschaft. Über die sozialen Netzwerke Twitter und Facebook habe ich mir neue Wahrnehmungskanäle eröffnet. Die bundesweit relevanten Presseorgane verfolge ich nicht weniger intensiv als früher. Meine Wahrnehmung politischer Prozesse auf der europäischen Ebene hat sich in der gleichen Zeit, aus naheliegenden Gründen, verdichtet. Sie konzentriert sich allerdings auf die europäischen Institutionen selbst, nicht auf die darin wirkenden nationalstaatlichen Akteure. Was ich an bundespolitischer Vernetzung aufgegeben habe, konnte ich teilweise durch europäische Vernetzung ersetzen. Meine Wahrnehmung europäischer Prozesse bleibt dennoch deutsch geprägt. Sie wird ergänzt durch die Beobachtung europäischer think tanks, durch Medien wie EurActiv oder Open Europe Press Summary und durch das elektronische Angebot von vier bis fünf internationalen Presseorganen. 
Verändert hat sich auch das Spektrum der Gegenstände und Prozesse, die ich wahrnehme. War ich in meinen Parteifunktionen generalistisch gefordert, bin ich heute in erster Linie Fach-Abgeordneter - mit generalistischen Ambitionen. Meine Wahrnehmung konzentriert sich stärker als früher auf bestimmte Themenbereiche, die meinen fachlichen Schwerpunkten entsprechen. Meine generalistische Neigung kommt vor allem zum Tragen, indem ich versuche, den Debatten über die notwendige Neubestimmung des europäischen Narrativs - heute erscheint die EU als Projekt ohne einen derartigen Leitfaden -, über die europäische Krise, common economic governance und einen Europäischen Green New Deal zu folgen oder mich aktiv an ihnen zu beteiligen.

Verändert hat sich schließlich neben Quellen und Gegenständen auch der Modus meiner Wahrnehmung. Sie ist heute deutlich stärker individualistisch geprägt. Was „für mich“ wichtig ist, hat an Bedeutung gewonnen gegenüber dem, was „für uns“ wichtig ist.

\section{Können Sie konkrete Ausprägungen dieser Individualisierung benennen?}

Drei Erfahrungen, die meinen Handlungsraum wesentlich bestimmen, drängen sich auf. Zum einen, dass ich, um mich als Fachpolitiker zu profilieren, eine gewisse Eigenständigkeit zeigen muss, wo ich vorher, zumal zu Regierungszeiten, Kooperation herzustellen hatte. Zweitens die Erfahrung, dass es innerhalb der eigenen Parteifamilie nicht immer sinnvoll ist, einen Konsens oder Kompromiss anzustreben, weil aufgrund unterschiedlicher politischer Kulturen und verschiedener Positionierungen im jeweiligen nationalen Diskurs mit einem Konsens oder Kompromiss etwa zwischen „meiner“ und einer „französischen“ Position in wichtigen Streit- und Identitätsfragen zuhause keinem wirklich gedient ist. Schließlich die Erfahrung, dass es im Europäischen Parlament möglich ist, dort, wo ich mit Abgeordneten anderer Fraktionen eventuell sogar mehr Gemeinsamkeiten erkenne als mit Kollegen der eigenen Fraktion, dies in praktisch relevantes Handeln, in wirksames gemeinsames Auftreten und sogar in erfolgreiche Mehrheitsbildung umzumünzen. In ihrer Gesamtheit führen diese Erfahrungen sowohl zu größerer Vereinzelung als auch zu mehr Freiraum.

Die Veränderung der konkreten Produktionsbedingungen meiner politischen Arbeit haben allerdings, so weit mir das bewusst ist, nicht dazu geführt, dass ich die Dinge „europäischer“ wahrnehme als früher. Ich beobachte an mir nicht den Reflex, regelmäßig ,als Europäer“ einem ,nationalen“ Standpunkt gegenüber zu treten. Im Gegenteil nehme ich eher kritischer als zuvor wahr, wie sehr ein sol- 
cher „europäischer“ Eigensinn der Entwicklung von ownership, von Verantwortungsgefühl für die europäischen Prozesse in den und durch die Mitgliedstaaten im Weg steht. Als Vertreter europäischer Interessen gegenüber der nationalen Ebene verstehe ich mich nicht. Die Hypostasierung eines europäischen Interesses halte ich für ein Missverständnis. Nach meinem Verständnis stehen sich nicht nationale und europäische Interessen gegenüber, sondern nationale und regionale Interessen sowie die Anliegen der Bürgerinnen und Bürger. Diese müssen europäisch zur Verständigung, zum Ausgleich gebracht werden.

Sehen Sie eine Möglichkeit, wie das „Auseinanderfallen“ beider Welten, der nationalstaatlichen und der europäischen, zugunsten einer genuin europäischen, aber mitgliedstaatlich vermittelten Politik überwunden oder zumindest moderiert werden könnte?

Ich behaupte, dass kein Ende dieses Übels, des Auseinanderfallens, absehbar ist, wenn nicht entweder die Europapolitikerinnen und Europapolitiker nationalstaatlich wirkende politische Akteure werden oder die nationalen politischen Akteure in verständiger und geeigneter Weise an der Europapolitik teilnehmen.

Wenige Europapolitikerinnen und Europapolitiker sind tatsächlich auch politische Akteure auf mitgliedstaatlicher Ebene. Welche Deutschen wären zu nennen? In der Grünen Fraktion Dany Cohn-Bendit, der diese Rolle viele Jahre gleich in zwei Ländern, in Frankreich und Deutschland, gespielt hat. In der EVPFraktion am ehesten Elmar Brok, früher mehr als heute. In der sozialdemokratischen Fraktion Martin Schulz. Lothar Bisky war ein nationaler politischer Akteur, bevor er nach Brüssel und Straßburg zog. Frau Koch-Mehrin hätte es werden wollen. Das war es schon.

Nationale politische Akteure können nur dann Europapolitiker werden, wenn sie zuhause Macht erwerben. Definitionsmacht, die durch Medien vermittelt wird, institutionelle Macht durch politische Ämter und starke Vernetzung. Europapolitiker im EP kämpfen zu wenig um solche Macht. Sie fühlen sich ,in Europa“ im Zentrum des Geschehens und ignorieren dabei, dass, verstärkt durch die derzeit erkennbare Welle geistiger Re-Nationalisierung, die durch die EU schwappt, entscheidende europapolitische Machtpotentiale nach wie vor in den Hauptstädten liegt.

Als die schwarz-gelbe Koalition 2009 ihren Koalitionsvertrag aushandelte, wurden die EP-Politiker jedenfalls auf der Seite der CDU/CSU beim europapolitischen Teil nicht einbezogen. Sie litten still und duldeten es. Als die Bundesver- 
sammlung zwischen Herrn Wulff und Herrn Gauck den Bundespräsidenten wählte, gehörte ihr nach meiner Erinnerung nur ein einziger Europaabgeordneter an. Man bemängelte das unter Kollegen und duldete es. Wie viele Europaabgeordnete sind wenigstens Mitglieder der erweiterten Parteivorstände? Bei den Grünen eine von vierzehn. Wie viele leiten Bezirks- oder gar Landesverbände ihrer Parteien? Doch wie soll man solche Ämter erwerben und ausfüllen, wenn der Sitzungsplan des Europäischen Parlaments zwölf Wochen in Straßburg und 30 Wochen in Brüssel pro Jahr vorsieht? Der Bundestag hat knapp mehr als die Hälfte der Sitzungswochen des Europäischen Parlaments. Man muss die europäischen Sitzungsverpflichtungen öfter einmal ignorieren, wenn man national eine institutionell gewichtigere Rolle spielen will. Schon die Teilnahme an den Sitzungen des Europaausschusses des Bundestages lässt sich mit dem Sitzungskalender des EP nur ab und zu zur Deckung bringen.

Auch der Erwerb von Definitionsmacht fällt im Europaparlament schwer, weil das EP kaum richtige Debatten kennt. Die heutigen Aussprachen sind in der Regel lose Folgen von Erklärungen. Allenfalls in den „Elefanten“-Runden der Fraktionsvorsitzenden, etwa zur Nachbetrachtung der Ergebnisse eines Europäischen Rates, kommen tatsächlich argumentative Gefechte zustande. Meine Redezeit im Plenum des Parlaments liegt bei durchschnittlich etwa anderthalb Minuten pro Auftritt. Weil ich fleißig bei Twitter Ausdruckskürze übe, gelingen mir in dieser Zeit manchmal ein bis zwei knappe Argumente. Denkwürdige Parlamentsdebatten habe ich in Straßburg oder bei Brüsseler „Mini-Plenarsitzungen” nicht erlebt. Kaum je wurde über europäische Parlamentsdebatten in den Medien mehr als kursorisch berichtet. Ich erlebe im europarlamentarischen Geschäftsgang einen Mangel an strategischer Perspektive und strategischer Synthese. Wo strategisches Handeln zu beobachten ist, da findet es eher in Grauzonen, in informellen Zusammenhängen statt als in den offiziellen, parlamentarischen Abläufen. An informellen Strukturen sind das Europaparlament und sein Umkreis reich. Nur indem ich mich an etlichen von diesen beteilige, kann ich ansatzweise strategisch wirksam werden. Aber es ist fast unmöglich, aus Grauzonen heraus öffentlich zu glänzen.

Lässt sich daraus ableiten, dass die notwendige Vermittlungsarbeit besser dem Europäischen Rat oder der Kommission überlassen werden sollte?

Nein. Die Vermutung, dass vielleicht die Vertreter des Europäischen Rates oder der Europäischen Kommission eher als die Europarlamentarier geeignet seien, 
das „Auseinanderfallen der zwei Welten“ zu überwinden, geht meiner Meinung nach fehl. Im Europäischen Rat wird in der Regel nationale Politik ,mal siebenundzwanzig“" gemacht, mit Vorliebe intergouvernemental. Der ständige Präsident des Rates ist eher dessen Diener als die Personifizierung seiner europäischen Würde. Die Kommission hingegen wurde durch den Lissabonner Vertrag geschwächt.

Die Kompetenzen des Europäischen Parlaments als genuin europäische Instanz hat der Reformvertrag auf dem Papier erheblich gestärkt. Aber paradoxerweise führt die innere Verfasstheit des Parlaments dazu, dass es ihm systematisch schwer fallen muss, seiner Rolle gerecht zu werden - sofern sich seine Mitglieder die notwendige Macht nicht in ihren jeweiligen Hauptstädten verschaffen. Wenn sie allerdings darum ringen, besorgen sie damit zugleich das Geschäft Europas.

An die nationalen Medien zu appellieren, dem Thema „Europa“ doch mehr Beachtung zu schenken, wird erst dann fruchten, wenn die Medien aus sachlichen Erwägungen nicht mehr umhin kommen, „Europa“ (genauer: dem Europäischen Parlament) mehr Bedeutung zuzumessen, weil sie sonst ihrer nationalen Aufgabe nicht mehr gerecht würden. Das Problem sind also nicht die Medien. Es ist die Politik.

Ein zweiter Ansatzpunkt gegen das „Auseinanderfallen der beiden Welten“ besteht darin, dass die Abgeordneten der mitgliedstaatlichen Parlamente in neuer Weise europapolitisch aktiv werden. Für diese Perspektive hat niemand mehr getan als das Bundesverfassungsgericht, das den Bundestag mehrfach, zuletzt und am massivsten im Lissabon-Urteil, mit der Nase drauf gestoßen hat, dass es eine Integrationsverantwortung gibt, der er sich nicht entziehen darf.

In dem Maße, in dem die Bundestagsabgeordneten dem Gebot des Verfassungsgerichtes - eigentlich: des Grundgesetzes! - gerecht werden, ändert sich die Chance für eine genuin europäische, aber mitgliedstaatlich vermittelte Politik fundamental. Zum Positiven. Nationale Parlamentarier, die unter Nutzung ihrer Beteiligungsmöglichkeiten im europäischen Prozess anfangen, entsprechend Macht auszuüben, können die Brücke zwischen „Brüssel“ und „uns“ wahrscheinlich noch besser schlagen als umgekehrt. Denn die nationalen Abgeordneten sind schon dort präsent, wo die europäischen erst noch ankommen müssten. Wenn mein Wahlkreis-Abgeordneter sich damit profiliert, zu für mich relevanten europapolitischen Fragen vernehmlich Position zu beziehen, dann wird das auch eher ein Thema für mich als Wähler oder „Wutbürger“. 
Worin läge das Interesse nationalstaatlicher Abgeordneter, sich konsequenter in europäische Fragen einzumischen?

Es wäre darin begründet, dass sie nur durch Einmischung darauf hoffen können, Handlungsspielräume für demokratisch legitimierte Politik zurückzugewinnen, die in vielen Bereichen, vor allem in ökonomischen Fragen, nur auf europäischer Ebene sinnvoll möglich ist. Anderenfalls würden sie zu Verlierern dieser Verschiebung.

Ideal wäre es, wenn sich die Anstrengungen beim Brückenbau, so gegenläufig und in Wettbewerb verflochten ihre Motive sind, doch gegenseitig unterstützen könnten. Das setzt auf beiden Seiten eine bewusste Entscheidung für eine Strategie der „Solidarität der Parlamente“ voraus. Diese Entscheidung müsste auf der Einsicht fußen, dass beide Seiten ohne solche Kooperation jeweils schwächer wären. An der Frage der parlamentarischen Kontrolle der Gemeinsamen Sicherheits- und Verteidigungspolitik nach dem Lissabon-Vertrag entwickelt sich leider gerade das Gegenteil - eine feindliche Konkurrenz der Parlamente, von Eifersucht geprägt. Dabei böte etwa das aufgrund allgegenwärtiger Haushaltsnöte aktuelle Thema einer gemeinsamen Nutzung militärischer Fähigkeiten die Gelegenheit, von zwei Seiten her ein dickes Brett erfolgreich zu durchbohren.

Auch die Zivilgesellschaft hat beim Brückenbau zwischen den zwei Welten eine Rolle zu spielen, eine doppelte sogar. Zum einen kann sie, hoffentlich bald, mit dem Instrument der Europäischen Bürgerinitiative die Einmischung in die eigenen, europäischen Angelegenheiten praktizieren, Themen auf die Tagesordnung setzen, denen die europäischen Institutionen zu wenig Beachtung schenken. Der Umstand, dass Europa seinen Bürgerinnen und Bürgern eine direkte Mitwirkungsmöglichkeit eröffnet, die auf Bundesebene fehlt, kann dabei besonders positiv wirken. Zum anderen aber kann die Zivilgesellschaft manchmal auch in der Gestalt einzelner Interessengruppen, wenn sie ihren Horizont erweitern und ihr Geschäft professioneller betreiben, ganz eigennützig, sehr gemeinschaftsstiftend wirken. Ein Beispiel: Edmund Stoiber hat mit seiner hochrangigen Gruppe von Bürokratieabbau-Experten einen Vorschlag erarbeitet, wie Klein- und Kleinstunternehmen in Europa im Bereich der Bilanzierungspflichten entlastet werden können. Das Europaparlament ist dem schließlich gefolgt. Jetzt ist die Sache im Rat blockiert, weil bestimmte Interessengruppen durch nationalstaatliche Hebel das aufhalten wollen, was sie im EP nicht aufhalten konnten. Wie wäre es, nationale mittelständische Verbände nutzten die Position des EP als Ausgangspunkt 
für eine konzentrierte Kampagne zur konkreten Entlastung von Kleinen und Mittleren Unternehmen? Das wäre aussichtsreich und es würde die zwei Welten einander näher bringen.

Erkennen Sie mit Blick auf die divergenten Wahrnehmungsmuster nationalstaatlicher und europäischer Politiker einen Unterschied zwischen den großen Parteigruppierungen im Europäischen Parlament?

Nein. Ich sehe innerhalb der Parteigruppierungen im Europäischen Parlament mindestens so große Unterschiede diesbezüglich wie zwischen ihnen. Das ist, meine ich, ein guter Befund. Denn es lässt hoffen, dass es Koalitionen quer zu den Grenzen zwischen den Parteifamilien geben kann, um nationalstaatliche und europäische Politiken in Richtung auf eine ever closer union mit einander zu verweben. Einschließlich, selbstverständlich, eines gemeinsamen europäischen Finanzministeriums, wie Herr Trichet es uns aufgegeben hat.

Wie verändert sich die Rolle der europäischen Parteifamilien und Parteiverbände? Welche Folgen für das Europäische Parlament und für nationalstaatliche politische Parteien sind absehbar?

Seit ich bei den Europäischen Grünen aktiv engagiert bin, etwa zwölf Jahre umfasst dieser Zeitraum, hat sich die Bedeutung dieser parteipolitischen Ebene enorm verändert. 1999 war die Europäische Grüne Föderation wenig beachtlich. Die Delegierten der Mitgliedsparteien kamen bestenfalls aus deren zweiter, öfter aus der dritten Reihe. Systematische programmatische Arbeit fand nicht statt. Die Föderation war ein Debattierklub. Um die vorhandenen programmatischen Konflikte nicht permanent eskalieren zu lassen, beschränkte man bei Positionsbestimmungen zu sensiblen Themen die demokratische Methode der Meinungsbildung zumeist durch ein nicht formal, aber de facto geltendes Konsensprinzip. Daneben gab es die gegenseitige Duldung wenig relevanter, für den nationalen Parteikonsum gedachter Resolutionen. Als deutscher Vertreter kämpfte ich jahrelang intensiv, um zu verhindern, dass Entscheidungen der Grünen Föderation irritierende Auswirkungen für die grüne Realpolitik in der deutschen Bundesregierung haben könnten.

2004 wurde bei einem Kongress in Rom die Grüne Föderation in eine Europäische Grüne Partei (EGP) umgewandelt. Diese führte in der Europawahl jenes Jahres dann gleich die erste gemeinsame, EU-weite Wahlkampagne, die je orga- 
nisiert wurde. Jenseits dieser gemeinsamen Kampagne war der Wechsel von der Föderation zur Partei im Wesentlichen deklamatorisch. Bei näherem Hinsehen funktionierte die gemeinsame Wahlkampagne - ihr Slogan lautete: „Du entscheidest!“" - vor allem aus zwei Gründen: Zum einen konzentrierte sie sich mehr auf gemeinsame Wahlkampfmittel, Plakate und so weiter, als auf Wahlaussagen. Letztere blieben, in einem kaum beachteten Manifest niedergeschrieben, reichlich vage. Zum anderen gab es die pragmatische Übereinkunft, dass diejenigen nationalen Parteien, die weniger vom Wert der Kampagne überzeugt waren, nur so weit mitmachten, wie sie jeweils sinnvoll fanden, ohne aber die Überzeugteren durch Einwände daran zu hindern, das gemeinsame Vorgehen zum Markenzeichen zu machen.

Seither ist das Maß der Verbindlichkeit der Kooperation in der EGP deutlich gewachsen. Seit 2007 werden auch kontroverse programmatische Beschlüsse und Positionsbeschreibungen in normaler demokratischer Mehrheitsbildung mit wechselnden Mehrheiten zu einem Ergebnis geführt. In der Regel sind die Mitgliedsparteien bei den halbjährlichen Ratssitzungen der EGP durch Vertreterinnen und Vertreter ihrer Führungen repräsentiert. Es kommt vermehrt vor, dass politische Karrieren in der EGP in politische Karrieren in nationalen Parteien münden. In einer aktuellen Satzungsdiskussion erhebt die EGP, die eigentlich als Tochter aller ihrer Mitgliedsparteien entstand, leise Ansprüche auf eine europäische Führungs- und sogar Aufsichtsrolle gegenüber den nationalstaatlichen Parteiorganisationen. Zugleich entsteht eine Diskussion, dass grüne Europaabgeordnete nicht nur gegenüber den Parteien verantwortlich seien, von denen sie aufgestellt wurden, sondern auch direkt gegenüber der EGP.

Der zweite gemeinsame grüne Europawahlkampf 2009 war in Kommunikationsformen, Plakatsprache und Slogans wieder nationaler, hatte aber mit dem Konzept des Green New Deal eine starke, gemeinsam konzeptionell entwickelte und gemeinsam forcierte Botschaft. Und er hatte ein Team von gemeinsam ausgerufenen Spitzenkandidaten. Dadurch war dieser Wahlkampf zwar bunter in den Formen, aber politisch deutlich integrierter. Mit Blick auf die Europawahl 2014 arbeitet die EGP bereits seit 2010 daran, noch einmal weitere Schritte zur Gemeinsamkeit zu machen. Unter anderem wird dazu eine aktive Unterstützung für den Aufbau der nationalen Parteien in solchen EU-Ländern organisiert, in denen Grüne bisher eher schwach aufgestellt sind.

Die Zusammenarbeit innerhalb der Grünen europäischen Parteifamilie wird gestärkt durch die Green European Foundation. Dass diese existiert und nationale Parteien darin unterstützt, zuhause eigene Parteistiftungen aufzubauen, hat die 
besondere Rolle der parteinahen Böll-Stiftung aus Deutschland innerhalb der Grünen Familie zwar nicht ausgehebelt, aber etwas relativiert.

Eine formale Verbindlichkeit haben programmatische Beschlüsse der EGP für die Mitgliedsparteien oder die Europa-Abgeordneten nicht. Aber ihr Einfluss wächst. Insgesamt lässt sich für den betrachteten Zeitraum eine deutliche Europäisierung Grüner Parteipolitik feststellen. Neue Dimensionen dieser Europäisierung treten in jüngster Zeit hinzu. Es gibt einerseits Ansätze zu stärkerer bilateraler Kooperation zwischen Mitgliedsparteien, jetzt zum ersten Mal auch zwischen deutschen und französischen Grünen, und andererseits gewisse embryonale Formen der Projekt-Zusammenarbeit zwischen Akteuren verschiedener europäischer Parteifamilien.

Das Europäische Parlament muss ebenso wie die nationalen Parteien auf diese Veränderungen reagieren. Aus Parlamentssicht erweisen sie sich durchaus als positiv, denn sie steigern die Zahl der europäischen beziehungsweise der europäisch vernetzten Akteure, und das stärkt die unitarische Perspektive. Ideal wäre es, wenn es zur Europawahl 2014 von den europäischen Parteien nominierte transnationale Listen gäbe, nach denen drei bis fünf Prozent der Europaabgeordneten gewählt würden. Das würde viel dazu beitragen, dem EP in ganz Europa Gesicht zu geben.

Medial führt das Europäische Parlament unverändert eine Schattenexistenz. Die Medienlandschaft ist nationalstaatlich geprägt. In ihr haben die Aspekte der Parlamentsarbeit, die über das Vertreten nationaler Interessen hinausgehen, geringe Resonanz. Der Mangel dauerhafter parlamentarisch-politischer Konfliktlinien erschwert die Entwicklung öffentlicher Aufmerksamkeit für das EP noch mehr. Wie soll sich das verändern?

Die nationale Medienstruktur ist zweifellos eine Hürde bei der Schaffung von Sichtbarkeit für das Europäische Parlament. Aber ihre Ablösung durch Medien, die sich an eine europäische Öffentlichkeit wenden, ist nicht zu erwarten. Zudem kann sich eine europäische Öffentlichkeit auch im Dialog zwischen nationalen Medien verschiedener Länder herausbilden. In der Zeit um den 15.03.2003, dem Tag der großen europäischen Demonstrationen gegen den Irakkrieg, gab es eine europäische Öffentlichkeit, die sich, Medienstruktur hin oder her, europaweit auseinandersetzte und in der scharf voneinander geschiedene europapolitische Alternativen verfochten wurden. Auf der einen Seite standen die europäischen Freunde Präsident Bushs, von José Manuel Barroso bis hin zu Václav Havel, auf 
der anderen die „Achse des Friedens“ und die kriegskritische Position. Solche Ereignisse bilden Ausnahmen. Doch die Tatsache, dass es auch seither mehrfach gesamteuropäische Kontroversen gab, in denen die Alternativen deutlich wurden, spricht dafür, dass mehr das Fehlen hinreichend klar heraus gearbeiteter politischer Konflikte im EP dessen unscharfes öffentliches Profil verursacht als die Medienstruktur selbst.

Die fast permanente Große Koalition im Europäischen Parlament entpolitisiert dessen Arbeit gewiss. Doch sprach in der Vergangenheit immer wieder ein guter Grund für ihre Perpetuierung: die Chance, auf diese Weise qualifizierte Mehrheiten im Gesetzgebungsverfahren sicherzustellen und damit das Gewicht des Parlaments gegenüber dem Rat und der Kommission zu sichern. Dieses institutionelle Argument wird sicher bleiben. Trotzdem böte der Parlamentsalltag weit mehr Chancen zum profilierten und profilierenden Konflikt. Das lässt sich bisher jedoch mit der Interessenlage der beteiligten Parteien erklären. Für die konservative Mehrheitsfraktion trägt die Große Koalition dazu bei, die eigene Dominanz sicherzustellen. Die kleinen Fraktionen sind noch zu schwach, um die Machtkalküle zu verändern. Deshalb hängt hier viel von der sozialdemokratischen Fraktion ab. Ihr Vorsitzender hat sich seinen Namen damit gemacht, dass er sich lustvoll in eine scharfe Kontroverse stürzte. Er weiß gewiss, was möglich wäre. Bisher war für seine Fraktion die Große Koalition schlicht ertragreicher. Doch das könnte sich in den kommenden Jahren ändern. Die Sozialdemokratie gerät nämlich von zwei Seiten her unter Druck, sich im Europäischen Parlament scharfkantiger zu präsentieren. Zum einen ist sie inzwischen im Europäischen Rat - vier von 27 Regierungschefs! - und in der Kommission - sechs von 27 Kommissaren! - nur noch so schwach präsent, dass sie ohne erhöhte Sichtbarkeit im Parlament gegenüber den Konservativen immer weniger europäische Prägekraft dokumentieren kann. Zum andern bedroht ein europäisches Anwachsen der Grünen als Konkurrenz Stück für Stück die progressive Vorherrschaft der Sozialdemokraten. Ohne ihr Profil zu erhöhen, können sie in einem hypothetischen Bündnis „links von der EVP“ nicht hegemonial bleiben. Es ist also durchaus Grund für die ironische Hoffnung, dass gerade aus linker Schwäche eine gewisse Politisierung entsteht, die aus Stärke nicht erwuchs.

Pro-europäische ad-hoc-Bündnisse im Europäischen Parlament - gegen die Untergrabung von Schengen, gegen Pressegesetze von Berlusconi oder Orbán, gegen Sarkozys Roma-Politik, gegen deutsche Blockaden einer wirksamen ökonomischen Kooperation - sind ein zweiter Ansatzpunkt für die Steigerung der Sichtbarkeit des Europäischen Parlaments, denn in solchen Konflikten bewährt 
es sich als Verteidiger der europäischen Verheißungen gegen rückwärtsgewandte nationalistische und chauvinistische Kräfte. Dazu muss das Parlament und müssen seine Fraktionen ihre Arbeit allerdings ändern. Sie müssen neben dem Arbeits-Parlament auch ein „Kampagnen-Parlament“ werden. Vor dem Hintergrund der aktuellen Krise sehe ich dafür einen wachsenden Resonanzboden.

Im Vorfeld der Europawahl 2014 erwarte ich also beides: mehr parteipolitische Kontroversen, die Profil schaffen, und mehr Initiativen als „KampagnenParlament". 